Review

\title{
Artificial intelligence in the diagnosis of COVID-19: challenges and perspectives
}

\author{
Shigao Huang1\#, Jie Yang2,3\#, Simon Fong ${ }^{2}$, Qi Zhao ${ }^{\boxplus}$ \\ 1. Cancer Centre, Institute of Translational Medicine, Faculty of Health Sciences, University of Macau 999078, Macau SAR, China. \\ 2. Department of Computer and Information Science, University of Macau 999078, Macau SAR, China. \\ 3. Chongqing Industry \& Trade Polytechnic 408000, Chongqing, China. \\ \# Shigao Huang and Jie Yang contributed equally to this work \\ $\bowtie$ Corresponding authors: Qi Zhao, qizhao@um.edu.mo; Simon Fong, ccfong@um.edu.mo
}

(C) The author(s). This is an open access article distributed under the terms of the Creative Commons Attribution License (https://creativecommons.org/licenses/by/4.0/). See http:/ /ivyspring.com/terms for full terms and conditions.

Received: 2021.01.30; Accepted: 2021.03.06; Published: 2021.04.10

\begin{abstract}
Artificial intelligence $(\mathrm{Al})$ is being used to aid in various aspects of the COVID-19 crisis, including epidemiology, molecular research and drug development, medical diagnosis and treatment, and socioeconomics. The association of $\mathrm{Al}$ and COVID-19 can accelerate to rapidly diagnose positive patients. To learn the dynamics of a pandemic with relevance to $\mathrm{Al}$, we search the literature using the different academic databases (PubMed, PubMed Central, Scopus, Google Scholar) and preprint servers (bioRxiv, medRxiv, arXiv). In the present review, we address the clinical applications of machine learning and deep learning, including clinical characteristics, electronic medical records, medical images (CT, X-ray, ultrasound images, etc.) in the COVID-19 diagnosis. The current challenges and future perspectives provided in this review can be used to direct an ideal deployment of Al technology in a pandemic.
\end{abstract}

Key words: Artificial intelligence, COVID-19, diagnosis, deep learning, machine learning

\section{Introduction}

Coronavirus disease 2019 (COVID-19) was firstly reported in December 2019 [1-3]. It has caused a large number of deaths and negatively impacted people's lives worldwide, with more than 100 million confirmed cases of the new coronavirus (SARS-Cov-2) and more than 200 million cumulative deaths worldwide as of late January 2021[4, 5]. The patients experience flu-like symptoms such as fever, dry cough, tiredness, difficulty breathing. In more severe cases, the SARS-CoV-2 infection often causes fatal pneumonia in the patients [6]. Although the rapid progress in vaccines, the epidemic continues to spread out more than 200 countries and regions. In some countries, people have to face new lifestyles to combat COVID-19 [7]. Therefore, there is still an imminent need to develop antiviral drugs and medical practices to cure COVID-19 patients [5]. Many researchers from all over the world are seeking the effective techniques to cope with such challenges $[8,9]$.

In severe infectious disease outbreaks, both hospitals and physicians are suffered with the increasing workloads that weaken their ability to classify and hospitalize the suspected patients. The previous reports indicated that some of patients with early coronaviral infection were negative for CT [10], limiting the ability of radiologists to reliably rule out disease. While waiting 4-48 hours for confirmation of SARS-CoV-2 coronavirus by RT-PCR, the infected patients may transmit the virus to the close contacts if insufficient resources are used to separate positive patients with other suspected cases. In one report, the hospital-acquired infection was inferred in approximately $40 \%$ of cases [11]. It is imperative to speedily confirm patients for COVID-19 because initial false negative cases may increase the risk of transmission of the virus to others.

Artificial intelligence (AI) has been deployed at various levels of the health care system, including diagnosis, public health, clinical decision making, and therapeutics $[12,13]$. Particularly, AI algorithm plays 
an important role in the fast detection of COVID-19 patients during the current pandemic [14]. The number of studies using AI techniques to diagnose COVID-19 rapidly increased in 2020. Most reviews focus on describing diagnosis of COVID-19 from chest CT images using AI technology [15]. Therefore, we will comprehensively review the applications of AI for rapid diagnosis of COVID-19 with different medical analyses as well as discuss their challenges and perspectives in COVID-19. As shown in Fig. 1, it is mainly compiled from two aspects: machine learning (ML) and deep learning (DL), including electronic medical records, and medical images (CT, X-ray, ultrasound images, etc.), in clinical COVID-19 diagnosis.

\section{Machine learning-based diagnostic applications}

The potential applications of ML for COVID-19 have been previously described [14, 16-26]. The details are summarized in Table 1.

The first priority of ML was suggested as technical support for early detection and diagnosis of infections. A recent study demonstrated that the more accurate diagnosis could be generated using a computational model trained on large clinical datasets [16]. An association between males and higher serum lymphocyte and neutrophil levels was identified by applying ML to reanalyze COVID-19 data from 151 published studies. The COVID-19 patients could be classified into three clinically relevant subtypes based on serum levels of immune cells, gender, and reported symptoms. A sensitivity of $92.5 \%$ and a specificity of $97.9 \%$ were achieved to discriminate COVID-19 patients from influenza patients using a computational classification model. another study reported that early identification could be performed by a ML model based on the clinical symptoms without CT images at the time of fever clinic admission [17]. Peng $M$ et al reported that 18 diagnostic indicators for COVID-19 were highly associated with a significant diagnosis of COVID-19 using AI screening, which improved the accuracy of the clinical diagnosis [18]. Chen et al described a ML random forest model used to classify COVID-19 clinical types, which achieved $>90 \%$ predictive accuracy [19]. Zoabi et al generated a ML model that trained on data from 51831 tested individuals in Israel. This model resulted in high accuracy using only eight binary features [20]. A combination of seven ML algorithm based on data from UCLA Health System in United States was established to diagnose COVID-19 in the inpatient setting [21]. In the test set $(n=392)$, the combined model achieved excellent diagnostic metrics compared to RT-PCR. An et al developed five ML algorithms for death prediction in a case dataset that was provided by the Korean National Health Insurance Service (KNHIS) [5]. In prediction of mortality, the sensitivity and specificity exceeded $90 \%$ while the areas under the curves (AUC) exceeded 96\%.

Chest CT has been used to evaluate the patients with suspected SARS-CoV-2 infection. AI system had equal sensitivity as compared to a senior thoracic radiologist. The radiologists with less expertise in chest imaging demand $\mathrm{AI}$-assisted screening. In a test set of 279 patients, an AI algorithm combining chest CT presentation correctly identified $68 \%$, while radiologists classified all of these patients as COVID-19 negative [14].

\section{Artificial intelligence for COVID-19 diagnosis}
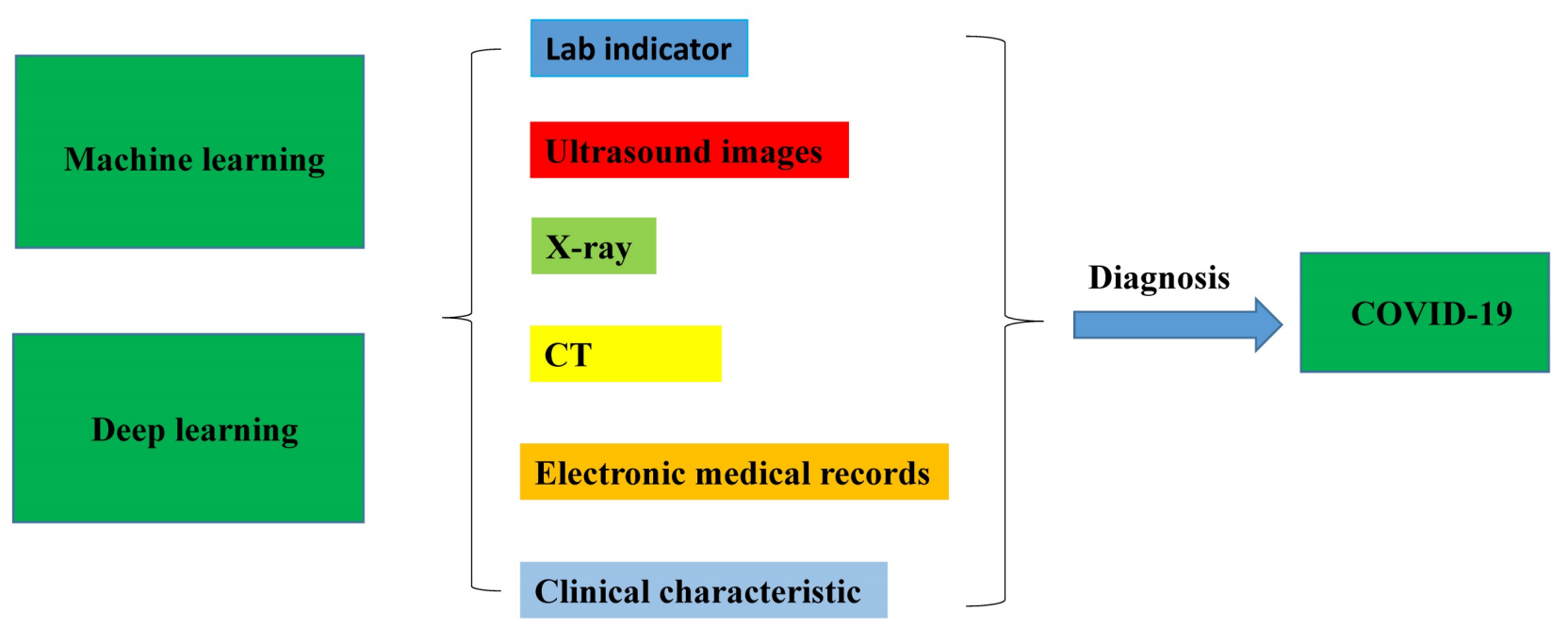

Figure 1. A flowchart of the artificial intelligence methods for COVID-19 diagnosis: machine learning and deep learning were applied in the medical characteristic to diagnosis the COVID-19 infection. 
Table 1. Application of machine learning-based COVID-19 diagnosis

\begin{tabular}{|c|c|c|c|c|c|}
\hline Authors & Countries & Data Sources & No. of Patients & Techniques & Performances \\
\hline An et al.[5] & Korea & KNHIS & 10237 & $\begin{array}{l}\text { LASSO, LSVM, } \\
\text { SVM with radial basis } \\
\text { function kernel, } \\
\text { RF, KNN }\end{array}$ & $\begin{array}{l}\text { Sensitivities }(90.7 \% \text { [95\% confidence interval: } 83.3,97.3] \\
\text { and } 92.0 \%[85.9,98.1], \text { respectively) } \\
\text { Specificities }(91.4 \%[90.3,92.5] \text { and } 91.8 \%,[90.7,92.9], \\
\text { respectively) } \\
\text { AUC }(0.963[0.946,0.979] \text { and } 0.962[0.945,0.979], \text { respectively) }\end{array}$ \\
\hline Zoabi et al.[20] & Israel & Israeli Ministry of Health & 8393 & $\begin{array}{l}\text { Gradient boosting with } \\
\text { decision tree }\end{array}$ & 0.90 auROC $95 \%$ CI: $0.892-0.905$ \\
\hline Batista et al.[23] & Brazil & Brazilian Ministry of Health & 235 & $\begin{array}{l}\text { Neural } \\
\text { networks,RF,LR,SVM, } \\
\text { Gradient boosting trees }\end{array}$ & $\begin{array}{l}\text { AUC: } 0.85 ; \\
\text { Sensitivity: } 0.68 \text {; } \\
\text { Specificity: } 0.85 ; \\
\text { Brier Score: } 0.16\end{array}$ \\
\hline Prefeitura et al.[24] & Brazil & $\begin{array}{l}\text { Public Health Department } \\
\text { of Florianópolis }\end{array}$ & 3916 & Random forest & $\begin{array}{l}\text { Accuracy:0.66 (UI 95\%0.62-0.69) } \\
\text { Sensitivity:0.65 (UI 95\%0.57-0.75); } \\
\text { Specificity:0.66 (UI 95\%0.60-0.70) }\end{array}$ \\
\hline Mei et al.[14] & China & $\begin{array}{l}18 \text { medical centers } \\
\text { in } 13 \text { provinces in China }\end{array}$ & 419 & CNN, SVM, RF, MLP & AUC 0.92 \\
\hline Chen et al.[19] & China & Union Hospital, Wuhan, China & 214 & RF & Accuracy $>95 \%$ \\
\hline Li et al.[16] & USA & Public data & 413 & XGBoost & $\begin{array}{l}\text { Sensitivity } 92.5 \% \\
\text { Specificity } 97.9 \%\end{array}$ \\
\hline Avila et al.[25] & Brazil & $\begin{array}{l}\text { Hospital Israelita Albert Einstein } \\
\text { (HIAE - São Paulo, Brazil) }\end{array}$ & 510 & Naïve Bayes Classifier & Sensitivity and Specificity $76.7 \%$ \\
\hline
\end{tabular}

Developed ML models are suitable for surveillance efforts to predict the SARS-CoV-2 infection risk of patients with severe diseases. Monahan et al reported that two unique ML models were generated to predict the risk of the hemodialysis (HD) patients having the undetected SARS-CoV-2 infections [27]. Through analyzing more than ten thousand patients, they identified top predictor of an HD patient having a SARS-CoV-2 infection in the prior week.

\section{Deep learning-based diagnostic applications}

DL, as a subset of $\mathrm{ML}$, has been explored extensively in the diagnosis of COVID-19, especially in the field of lung detection images, including CT images, X-ray images and ultrasound images. The details are summarized in Table 2.

$\mathrm{Wu}$ et al developed a DL-based screening framework for coronavirus through a multi-view chest CT imaging [28]. The framework trained on multi-view images of chest $\mathrm{CT}$ images from two different hospitals in China through a Convolutional Neural Network (CNN) variant, ResNet 50, with a total dataset of 495 patient images, including 368 confirmed cases and 127 images of suspected pneumonia cases. Based on DL method, the proposed diagnosis model showed $76 \%$ accuracy, $81.1 \%$ sensitivity, $61.5 \%$ specificity, and $81.9 \%$ AUC. Ardakani et al developed a variant system based on $\mathrm{CT}$ images in combination with the CNN architectures for COVID-19 diagnostic detection in which ten convolutional neural networks were used to discriminate positive COVID-19 infections from non-infection groups. Among all networks,
ResNet-101 showed the best performance with 99.51\% accuracy, 100\% sensitivity, 99.4\% AUC, and 99.02\% specificity [29]. Cifci et al diagnosed infections from CT images used AlexNet and Inception-V4, pre-trained models that are widely used in medical image analysis [30]. The dataset consists of a public database of 5800 CT images (4640 CT images, with 4640 training samples and 1160 test sets). The dataset analysis has shown a sensitivity of $94.74 \%$ and a specificity of $87.37 \%$.

Hybrid systems show the higher accuracy than a single model. Hasan et al generated a hybrid system that combined Q-deformed entropy and DL features (QDE-DF) with LSTM from extracting deep features of CT images [31]. The dataset contains 321 chest CT samples, including 118 of COVID-19 cases images, 96 images of pneumonia cases, and 107 images of healthy individuals. As the result, 16 core attributes were extracted by the proposed composite model. The analysis achieved $99.68 \%$ accuracy in the training and test sets in the ratio of 7:3.

Multitask DL based model can be used to detect COVID-19 lesions on CT scans. A multitask DL model, including segmentation, classification and reconstruction, was used to detect COVID-19 patient and segment COVID-19 lesion from chest CT images [32]. The proposed model was used to analyze a dataset of 1369 patients including 449 patients with COVID-19, 425 healthy individuals, 98 with lung cancer and 397 cases of other diseases. The model had an accuracy of $86 \%$, a sensitivity of $94 \%$, a specificity of $79 \%$, and an AUC of $93 \%$.

Moreover, transfer learning has been applied for the early diagnosis of coronaviruses based on X-ray imaging. Apostolopoulos and Bessiana proposed a 
system for the automatic diagnosis of COVID-19 cases in which five CNN variants (VGG19, MobileNetv2, Inception, Xception, and Inception-ResNetv2) were used to analyze a dataset of X-ray images from patients with common bacterial pneumonia, confirmed Covid-19 disease, and normal incidents [33]. The dataset analysis suggests that DL with X-ray imaging has a high accuracy, sensitivity, and specificity $(96.78 \%, 98.66 \%$, and $96.46 \%$, respectively). A generative adversarial network (GAN) with deep transfer learning has been proposed for coronavirus detection in chest $X$-ray images [34]. The total number of X-ray images in the collection was 307 and contained four categories: COVID-19, normal, pneumonia bacteria, and pneumonia virus. The models contain the Alexnet, Googlenet, and Restnet18. The accuracy of selecting Alexnet as the primary deep transfer model can reach $80.6 \%$ when four categories are included, while the accuracy of selecting Googlenet as the primary model can reach $85.2 \%$ when three classes are included. Another COVID-19 diagnosis-Net based on an X-ray image was proposed by Ucar and Korkmaz [35]. Image data from three public datasets obtained $98.26 \%$ accuracy, $98.25 \%$ specificity, and $97.39 \%$ F1-score in the proposed system. In another study, a DarkNet model for automatic COVID-19 detection using chest X-ray images has been developed [36]. The DarkNet model is a classifier used as a "you only look-once" (YOLO) real-time object detection system. The proposed model was evaluated for binary classification (COVID vs. No-Findings, the classification accuracy of $98.08 \%$ ) and multi-class classification (COVID vs. No-Findings vs. Pneumonia. It has shown a classification accuracy of $98.08 \%$. In addition, the generated heatmaps can assist the clinicians to locate the affected regions on chest X-rays.

Table 2. Application of deep learning-based COVID-19 diagnosis

\begin{tabular}{|c|c|c|c|c|c|c|c|}
\hline Authors & Data Sources & No. of Images & $\begin{array}{l}\text { Type of } \\
\text { Images }\end{array}$ & No. of Classes & Techniques & Type of model & Performances \\
\hline $\begin{array}{l}\text { Ardakani et } \\
\text { al.[29] }\end{array}$ & $\begin{array}{l}\text { Real-time data from } \\
\text { university hospital }\end{array}$ & $\begin{array}{l}1020(\text { COVID19=510, } \\
\text { non-COVID19=510) }\end{array}$ & CT & $\begin{array}{l}2 \text { (COVID-19, } \\
\text { non-COVID19) }\end{array}$ & $\begin{array}{l}\text { AlexNet, VGG16, } \\
\text { VGG-19, } \\
\text { SqueezeNet, } \\
\text { GoogleNet, } \\
\text { MobileNet-V2, } \\
\text { ResNet-18, ResNet-50, } \\
\text { ResNet-101, Xception }\end{array}$ & Pre-trained model & $\begin{array}{l}\text { Accuracy }=99.51, \\
\text { Sensitivity=100, } \\
\text { Specificity=99.02, } \\
\text { Precision=99.27, } \\
\text { AUC }=99.4, \\
\text { NPV }=100\end{array}$ \\
\hline Wu et al. [28] & $\begin{array}{l}\text { China Medical University, } \\
\text { Beijing Youan Hospital }\end{array}$ & $\begin{array}{l}495(\text { COVID19=368, other } \\
\text { pneumonia }=127)\end{array}$ & CT & $\begin{array}{l}2 \text { (COVID-19, other } \\
\text { pneumonia) }\end{array}$ & ResNet50 & Pre-trained model & $\begin{array}{l}\text { Accuracy }=76, \\
\text { Sensitivity=81.1, } \\
\text { Specificity }=61.5, \\
\text { AUC }=81.9\end{array}$ \\
\hline Cifci [30] & $\begin{array}{l}\text { kaggle.com (benchmark } \\
\text { web of dataset science) }\end{array}$ & 5800 & CT & $\begin{array}{l}2 \text { (COVID-19, other } \\
\text { pneumonia) }\end{array}$ & AlexNet, Inception-V4 & Pre-trained model & $\begin{array}{l}\text { Accuracy }=94.74, \\
\text { Sensitivity }=87.37, \\
\text { Specificity }=87.45\end{array}$ \\
\hline $\begin{array}{l}\text { Apostolopoulos } \\
\text { and Bessiana } \\
\text { [33] }\end{array}$ & $\begin{array}{l}\text { COVID-19 X-ray image } \\
\text { database[48], Kaggle } \\
\text { dataset }^{*},\end{array}$ & $\begin{array}{l}1442(\text { COVID19=224, } \\
\text { pneumonia }=714, \\
\text { normal }=504)\end{array}$ & X-RAY & $\begin{array}{l}3 \text { (COVID-19, pneumonia, } \\
\text { normal) }\end{array}$ & $\begin{array}{l}\text { VGG19, MobileNetv2, } \\
\text { Inception, Xception, } \\
\text { InceptionResNetv2 }\end{array}$ & Pre-trained model & $\begin{array}{l}\text { Accuracy }=96.78, \\
\text { Sensitivity }=98.66, \\
\text { Specificity }=96.46\end{array}$ \\
\hline Loey et al. [34] & $\begin{array}{l}\text { COVID-19 X-ray image } \\
\text { database[48], Dataset* }\end{array}$ & $\begin{array}{l}307 \\
(\mathrm{COVID}=69, \text { normal=79, } \\
\text { pneumonia_bac } \\
=79, \\
\text { pneumonia_vir= } \\
79)\end{array}$ & X-RAY & $\begin{array}{l}4 \text { (COVID, normal, } \\
\text { pneumonia_ba, } \\
\text { pneumonia_vir) }\end{array}$ & $\begin{array}{l}\text { GAN, Alexnet, } \\
\text { Googlenet, Resnet18 }\end{array}$ & Pre-trained model & $\begin{array}{l}\text { Accuracy }=85.2, \\
\text { Precision }=80.6,\end{array}$ \\
\hline Hasan et al.[31] & $\begin{array}{l}\text { COVID-19 Dataset*, } \\
\text { SPIEAAPM-NCI Lung } \\
\text { Nodule } \\
\text { Classification Challenge } \\
\text { Dataset }\end{array}$ & $\begin{array}{l}321(\text { COVID19=118, } \\
\text { pneumonia }=96, \\
\text { healthy=107) }\end{array}$ & CT & $\begin{array}{l}3 \text { (COVID19, pneumonia, } \\
\text { healthy) }\end{array}$ & QDE-DF & Customized Model & Accuracy $=99.68$ \\
\hline $\begin{array}{l}\text { Amyar et al. } \\
{[32]}\end{array}$ & $\begin{array}{l}\text { COVID-CT[49], } \\
\text { COVID-19 CT } \\
\text { segmentation dataset*, a }^{*} \text { hospital named Henri } \\
\text { Becquerel Center }\end{array}$ & $\begin{array}{l}1044(\text { COVID19=449, } \\
\text { nonCOVID-19=595) }\end{array}$ & CT & $\begin{array}{l}2 \text { (COVID19, } \\
\text { nonCOVID-19) }\end{array}$ & $\begin{array}{l}\text { EncoderDecoder with } \\
\text { multi-layer } \\
\text { perceptron }\end{array}$ & Customized Model & $\begin{array}{l}\text { Accuracy }=86, \\
\text { Sensitivity }=94, \\
\text { Specificity }=79, \\
\text { AUC }=93\end{array}$ \\
\hline $\begin{array}{l}\text { Ozturk et } \\
\text { al.[36] }\end{array}$ & $\begin{array}{l}\text { COVID-19 X-ray image } \\
\text { database [48], ChestX-ray8 } \\
{[50]}\end{array}$ & $\begin{array}{l}1127(\mathrm{COVID}=127, \\
\text { no-finding }=500, \\
\text { pneumonia }=500)\end{array}$ & X-RAY & $\begin{array}{l}3 \text { (COVID, nofinding, } \\
\text { pneumonia) }\end{array}$ & DarkNet & Customized Model & $\begin{array}{l}\text { Accuracy }=98.08, \\
\text { Sensitivity }=95.13, \\
\text { Specificity }=95.3, \\
\text { Precision }=98.03, \\
\text { F1-Score }=96.51\end{array}$ \\
\hline $\begin{array}{l}\text { Rahimzade } \\
\text { h and Attar } \\
{[35]}\end{array}$ & $\begin{array}{l}\text { COVID-19 X-ray image } \\
\text { database [48], RSNA } \\
\text { Pneumonia Detection } \\
\text { Challenge dataset [51] }\end{array}$ & $\begin{array}{l}15085(\text { COVID19=180, } \\
\text { pneumonia }= \\
6054, \text { normal }=8851)\end{array}$ & X-RAY & $\begin{array}{l}3 \text { (COVID-19, pneumonia, } \\
\text { normal) }\end{array}$ & $\begin{array}{l}\text { Concatenated } \\
\text { CNN }\end{array}$ & Customized Model & $\begin{array}{l}\text { Accuracy }=99.50, \\
\text { Sensitivity }=80.53, \\
\text { Specificity }=99.56\end{array}$ \\
\hline
\end{tabular}

*Kaggle dataset: https://www.kaggle.com/andrewmvd/convid19-x-rays,

Dataset: https://drive.google.com/uc?id=1coM7x3378fOu216Pg2wldaOI7Dntu1a,

Covid-19 Dataset: https://radiopaedia.org,

Archive, C.I. SPIE-AAPM-NCI Lung Nodule Classification Challenge Dataset: https:/ / www.cancerimagingarchive.net,

COVID-19 CT segmentation dataset: http://medicalsegmentation.com/covid19/ .Note: CT: computerized tomography; CNN: Convolutional Neural Network 
Table 3. Challenges and perspectives of machine learning-based COVID-19 diagnosis

\begin{tabular}{ll}
\hline Challenges & Perspectives \\
\hline $\begin{array}{l}\text { Improve the } \\
\text { accuracy of the AI } \\
\text { diagnosis }\end{array}$ & $\begin{array}{l}\text { Combine chest imaging with clinical symptoms, exposure } \\
\text { history, and laboratory tests in the diagnosis of COVID-19 }\end{array}$ \\
$\begin{array}{l}\text { Reduce the false } \\
\text { negative diagnosis } \\
\text { rate }\end{array}$ & $\begin{array}{l}\text { Provides spare capacity for CT and X-ray imaging scans } \\
\text { with the advantages of rapid COVID-19 diagnosis. }\end{array}$ \\
\hline
\end{tabular}

Lung Ultrasonography (LUS) has been used for the detection and management of acute respiratory disorders. A recent study shows that DL techniques may assist clinician for the analysis of LUS images from COVID-19 patients [37]. A model with three different tasks on LUS imaging: frame-based classification, video-level grading and pathological artifact segmentation, was proposed in the diagnosis of COVID-19. It demonstrated accurate prediction and localization of LUS imaging biomarkers in COVID-19 patients.

\section{Challenges and perspectives}

AI has great potential and opportunity for rapid analysis of large amounts of data. It has played an important role in the prevention of COVID-19 outbreak [38]. AI models may be as accurate as experienced radiologists to diagnose COVID-19 [38].

It is noteworthy that although some patients infected with COVID-19 are asymptomatic, they have the potential to become transmitters of the virus [6, 39]. Although the infection can be confirmed by a polymerase chain reaction, COVID-19 patients with pneumonia symptoms may show a pattern on chest X-ray or CT images that are only moderately characteristic for the clinicians [40]. It is difficult to find people who are currently infected with COVID-19 but are asymptomatic [41]. The transmission rate of COVID-19 is determined with the ability to confidently recognize infected patients with low false-negative rates. Meanwhile, an effective control of false positives can avoid unnecessary quarantine of patients and thus further reduce the burden on the health care system.

Biomedical imaging (chest X-ray, CT scan, and ultrasound images, etc.) enables to visualize symptoms of pneumonia. Image processing techniques are attractive in the areas of biomedicine and cancer diagnosis [42]. It is well known that AI-based biomedical image diagnosis has achieved remarkable success. ML and DL methods have become valuable tools for the discovery of various diseases [40, 43-45]. For example, although some patients have already infected by SARS-Cov-2, they show the normal chest CT images. Therefore, the negative predictive value of chest CT images is limited and does not completely rule out infection. The accuracy of the solo AI diagnosis is still challenged. Therefore, to meet clinical needs, AI algorithms is required to combine chest imaging with clinical symptoms, exposure history, and laboratory tests in the diagnosis of COVID-19.

False negative rate is usually high with the laboratory tests, such as nucleotide RT-PCT [46]. Medical image screening can provide an intuitive and accurate diagnosis when it is used as the assisted testing method for COVID-19 [47]. In some epidemic countries, such China and United States, the AI model to diagnose negative sensitivity with $\mathrm{CT}$ has been used in the early infections [38]. This new strategy provides spare capacity for $\mathrm{CT}$ and X-ray imaging scans with the advantages of rapid COVID-19 diagnosis.

\section{Conclusion}

AI model may be as accurate as experienced physicians at diagnosing COVID-19. In this review, we discuss the challenges and perspectives of ML and DL in the COVID-19 as well as the need for further research. The clinical application of $\mathrm{AI}$ in the diagnosis of COVID-19 is promising, and additional extensive research is required.

\section{Acknowledgments}

The study is supported by the Science and Technology Development Fund, Macau SAR (File no. FDCT/0015/2018/A1， FDCT/126/2014/A3), the National Key R\&D Program of China (2019YFA0904400), the Multi-Year Research Grant of University of Macau (File no. MYRG2019-00069-FHS, MYRG2016-00069-FST), Guangzhou Science and Technology Innovation and Development of Special Funds (201807010004, EF003/FST-FSJ/2019/GSTIC, and EF004/FST-FSJ/2019/GSTI), National Natural Science Foundation of China (31440041), the Project of the Science and Technology Research Program of Chongqing Municipal Education Commission of China (File no. KJQN202003601, KJZD-K201903601).

\section{Author Contributions}

$\mathrm{SH}$ and JY contributed equally to this paper. $\mathrm{SH}$, JY, SF, and QZ conceived the paper. SH and JY wrote the initial draft and edits. All authors approved the final draft of the manuscript. QZ and SF are the supervisors. The corresponding authors are responsible that all listed authors meet authorship criteria.

\section{Competing Interests}

The authors have declared that no competing interest exists. 


\section{References}

1. Zhou G, Zhao Q. Perspectives on therapeutic neutralizing antibodies against the Novel Coronavirus SARS-CoV-2. Int J Biol Sci. 2020; 16: 1718-23.

2. Yi Y, Lagniton PNP, Ye S, Li E, Xu RH. COVID-19: what has been learned and to be learned about the novel coronavirus disease. Int J Biol Sci. 2020; 16: $1753-66$.

3. Deng CX. The global battle against SARS-CoV-2 and COVID-19. Int J Biol Sci. 2020; 16: 1676-7.

4. Sohrabi C, Alsafi Z, O'Neill N, Khan M, Kerwan A, Al-Jabir A, et al. World Health Organization declares global emergency: A review of the 2019 novel coronavirus (COVID-19). International journal of surgery. 2020; 76: 71-6.

5. Organization WH. Coronavirus disease 2019 (COVID-19): situation report, 82. 2020.

6. Huff HV, Singh A. Asymptomatic transmission during the coronavirus disease 2019 pandemic and implications for public health strategies. Clinical Infectious Diseases. 2020; 71: 2752-6.

7. Bhattacharya S, Maddikunta PKR, Pham Q-V, Gadekallu TR, Chowdhary CL, Alazab M, et al. Deep learning and medical image processing for coronavirus (COVID-19) pandemic: A survey. Sustainable cities and society. 2020; 65: 102589.

8. Fanelli D, Piazza F. Analysis and forecast of COVID-19 spreading in China, Italy and France. Chaos, Solitons \& Fractals. 2020; 134: 109761.

9. Kucharski AJ, Russell TW, Diamond C, Liu Y, Edmunds J, Funk S, et al. Early dynamics of transmission and control of COVID-19: a mathematical modelling study. The lancet infectious diseases. 2020; 20: 553-8.

10. Chung M, Bernheim A, Mei X, Zhang N, Huang M, Zeng X, et al. CT imaging features of 2019 novel coronavirus (2019-nCoV). Radiology. 2020; 295: 202-7.

11. Wang $Y$, Wang $Y$, Chen $Y$, Qin $Q$. Unique epidemiological and clinical features of the emerging 2019 novel coronavirus pneumonia (COVID-19) implicate special control measures. Journal of medical virology. 2020; 92: 568-76.

12. Chen J, See KC. Artificial Intelligence for COVID-19: Rapid Review. J Med Internet Res. 2020; 22: e21476.

13. Vaishya R, Javaid M, Khan IH, Haleem A. Artificial Intelligence (AI) applications for COVID-19 pandemic. Diabetes Metab Syndr. 2020; 14: 337-9.

14. Mei X, Lee H-C, Diao K-y, Huang M, Lin B, Liu C, et al. Artificial intelligence-enabled rapid diagnosis of patients with COVID-19. Nature medicine. 2020; 26: 1224-8.

15. Ozsahin I, Sekeroglu B, Musa MS, Mustapha MT, Uzun Ozsahin D. Review on Diagnosis of COVID-19 from Chest CT Images Using Artificial Intelligence. Comput Math Methods Med. 2020; 2020: 9756518.

16. Li WT, Ma J, Shende N, Castaneda G, Chakladar J, Tsai JC, et al. Using machine learning of clinical data to diagnose COVID-19: a systematic review and meta-analysis. BMC medical informatics and decision making. 2020; 20: 1-13.

17. Feng $C$, Huang $Z$, Wang $L$, Chen $X$, Zhai $Y$, Zhu F, et al. A novel triage tool of artificial intelligence assisted diagnosis aid system for suspected COVID-19 pneumonia in fever clinics. 2020.

18. Peng M, Yang J, Shi Q, Ying L, Zhu H, Zhu G, et al. Artificial intelligence application in COVID-19 diagnosis and prediction. 2020.

19. Chen Y, Ouyang L, Bao FS, Li Q, Han L, Zhu B, et al. An interpretable machine learning framework for accurate severe vs non-severe covid-19 clinical type classification. Available at SSRN 3638427. 2020.

20. Zoabi Y, Deri-Rozov S, Shomron N. Machine learning-based prediction of COVID-19 diagnosis based on symptoms. npj Digital Medicine. 4: 1-5.

21. Goodman-Meza D, Rudas A, Chiang JN, Adamson PC, Ebinger J, Sun N, et al. A machine learning algorithm to increase COVID-19 inpatient diagnostic capacity. Plos one. 2020; 15: e0239474.

22. Hasan AM, Al-Jawad MM, Jalab HA, Shaiba H, Ibrahim RW, AL-Shamasneh AaR. Classification of covid-19 coronavirus, pneumonia and healthy lungs in ct scans using q-deformed entropy and deep learning features. Entropy. 2020; 22: 517.

23. de Moraes Batista AF, Miraglia JL, Donato THR, Chiavegatto Filho ADP. COVID-19 diagnosis prediction in emergency care patients: a machine learning approach. medRxiv. 2020.

24. Garcia LP, Goncalves AV, de Andrade MP, Pedebos LA, Vidor AC, Zaina R, et al. Estimating underdiagnosis of covid-19 with nowcasting and machine learning: Experience from brazil. medRxiv. 2020.

25. Avila E, Kahmann A, Alho C, Dorn M. Hemogram data as a tool for decision-making in COVID-19 management: applications to resource scarcity scenarios. PeerJ. 2020; 8: e9482.

26. Brinati D, Campagner A, Ferrari D, Locatelli M, Banfi G, Cabitza F. Detection of COVID-19 infection from routine blood exams with machine learning: a feasibility study. Journal of medical systems. 2020; 44: 1-12.

27. Monaghan C, Larkin JW, Chaudhuri S, Han H, Jiao Y, Bermudez KM, et al. Artificial intelligence for covid-19 risk classification in kidney disease: Can technology unmask an unseen disease? medRxiv. 2020.

28. Wu X, Hui H, Niu M, Li L, Wang L, He B, et al. Deep learning-based multi-view fusion model for screening 2019 novel coronavirus pneumonia: a multicentre study. European Journal of Radiology. 2020; 128: 109041.

29. Ardakani AA, Kanafi AR, Acharya UR, Khadem N, Mohammadi A. Application of deep learning technique to manage COVID-19 in routine clinical practice using CT images: Results of 10 convolutional neural networks. Computers in Biology and Medicine. 2020;121: 103795.

30. Cifci MA. Deep learning model for diagnosis of corona virus disease from CT images. Int J Sci Eng Res. 2020; 11: 273-8.

31. Hasan AM, Al-Jawad MM, Jalab HA, Shaiba H, Ibrahim RW, Al-Shamasneh AR. Classification of Covid-19 Coronavirus, Pneumonia and Healthy Lungs in CT Scans Using Q-Deformed Entropy and Deep Learning Features. Entropy (Basel). 2020; 22.

32. Amyar A, Modzelewski R, Li H, Ruan S. Multi-task deep learning based CT imaging analysis for COVID-19 pneumonia: Classification and segmentation. Computers in Biology and Medicine. 2020; 126: 104037.

33. Apostolopoulos ID, Mpesiana TA. Covid-19: automatic detection from $x$-ray images utilizing transfer learning with convolutional neural networks. Physical and Engineering Sciences in Medicine. 2020; 43: $635-40$.

34. Loey M, Smarandache F, M Khalifa NE. Within the lack of chest COVID-19 X-ray dataset: a novel detection model based on GAN and deep transfer learning. Symmetry. 2020; 12: 651.

35. Rahimzadeh M, Attar A. A modified deep convolutional neural network for detecting COVID-19 and pneumonia from chest X-ray images based on the concatenation of Xception and ResNet50V2. Informatics in Medicine Unlocked. 2020; 19: 100360.

36. Ozturk T, Talo M, Yildirim EA, Baloglu UB, Yildirim O, Acharya UR. Automated detection of COVID-19 cases using deep neural networks with X-ray images. Computers in biology and medicine. 2020; 121: 103792.

37. Roy S, Menapace W, Oei S, Luijten B, Fini E, Saltori C, et al. Deep learning for classification and localization of COVID-19 markers in point-of-care lung ultrasound. IEEE Transactions on Medical Imaging. 2020; 39: 2676-87

38. Mei X, Lee H-C, Diao K-y, Huang M, Lin B, Liu C, et al. Artificial intelligence-enabled rapid diagnosis of patients with COVID-19. Nature Medicine. 2020; 26: 1224-8.

39. Day M. Covid-19: identifying and isolating asymptomatic people helped eliminate virus in Italian village. BMJ: British Medical Journal (Online). 2020; 368.

40. Huang S, Yang J, Fong S, Zhao Q. Artificial intelligence in cancer diagnosis and prognosis: Opportunities and challenges. Cancer Letters. 2020; 471: 61-71.

41. Bastolla U. How lethal is the novel coronavirus, and how many undetected cases there are? The importance of being tested. medRxiv. 2020.

42. Khan FA, Asif M, Ahmad A, Alharbi M, Aljuaid H. Blockchain technology, improvement suggestions, security challenges on smart grid and its application in healthcare for sustainable development. Sustainable Cities and Society. 2020; 55: 102018.

43. Yang J, Huang S, Tang R, Hu Q, Lan K, Wang H, et al. Broad Learning with Attribute Selection for Rheumatoid Arthritis. 2020 IEEE International Conference on Systems, Man, and Cybernetics (SMC): IEEE; 2020. p. 552-8.

44. Huang S, Yang J, Fong S, Zhao Q. Mining Prognosis Index of Brain Metastases Using Artificial Intelligence. Cancers. 2019; 11: 1140.

45. Peng M, Huang S, Lv Q, Chen C, Qin J, He M, et al. Distribution of ABO Blood Groups and Their Association With Low Risk of Severe COVID-19 Infection in Patients. Research Square; 2021. DOI: 10.21203/rs.3.rs139563/v1.

46. Li Z, Yi Y, Luo X, Xiong N, Liu Y, Li S, et al. Development and clinical application of a rapid IgM-IgG combined antibody test for SARS-CoV-2 infection diagnosis. Journal of Medical Virology. 2020; 92: 1518-24.

47. Giri B, Pandey S, Shrestha R, Pokharel K, Ligler FS, Neupane BB. Review of analytical performance of COVID-19 detection methods. Analytical and Bioanalytical Chemistry. 2021; 413: 35-48.

48. Cohen JP, Morrison P, Dao L, Roth K, Duong TQ, Ghassemi M. Covid-19 image data collection: Prospective predictions are the future. arXiv preprint arXiv:200611988. 2020

49. Xie X, Zhong Z, Zhao W, Zheng C, Wang F, Liu J. Chest CT for typical coronavirus disease 2019 (COVID-19) pneumonia: relationship to negative RT-PCR testing. Radiology. 2020; 296: E41-E5. 
50. Wang X, Peng Y, Lu L, Lu Z, Bagheri M, Summers RM. Chestx-ray8: Hospital-scale chest $x$-ray database and benchmarks on weakly-supervised classification and localization of common thorax diseases. Proceedings of the IEEE conference on computer vision and pattern recognition; 2017. p. 2097-106.

51. Abbas A, Abdelsamea MM, Gaber MM. Classification of COVID-19 in chest X-ray images using DeTraC deep convolutional neural network. Applied Intelligence. 2020: 1-11. 